\title{
Modalidad virtual de enseñanza-aprendizaje: ventajas y desventajas
}

\author{
Sandra Milena Chicas Sierra9 \\ Sebastián Chacón Marín ${ }^{10}$
}

\section{Introducción}

$\mathrm{E}$ l proceso de globalización trajo consigo muchos cambios a nivel mundial. Uno de estos fue la manera en que se comunican las personas hoy en día. Gracias a los avances en la tecnología de la información y las comunicaciones, las personas cuentan con herramientas como el computador y el Internet, que facilitan la forma en que los seres se relacionan entre sí. El campo de la educación no se quedó atrás en este proceso, ya que, con el desarrollo de las TIC se han creado las llamadas herramientas de enseñanza- aprendizaje virtual.

Pinto (2006), aclara que la tecnología va a funcionar como herramienta de la imaginación y la construcción, y no como fin en sí misma y Litwin (2005), menciona que las tecnologías proveen formas de representación diferentes de las que puede simplemente proporcionar el docente, ayudando o enriqueciendo las comprensiones.

Igualmente, el uso de plataformas virtuales puede llegar a facilitar las tareas de enseñanza para los docentes, quienes a través del llamado campus virtual tienen la oportunidad de interactuar con los estudiantes de muchas partes de la ciudad y del mundo. Entendiendo por campus virtuales, estructuras creadas a manera de comunidad virtual en la que se desarrollan diversas actividades académicas de una institución educativa (Mansilla y Ugliarolo, 2013).

Según Rodríguez (2009), la plataforma virtual engloba un amplio rango de aplicaciones informáticas instaladas en un servidor cuya función es la de facilitar al profesorado la creación, administración, gestión y distribución de cursos por medio de Internet. Las plataformas virtuales incluyen herramientas como foros, chats, teleconferencias o e-mails

\footnotetext{
9. Docente universitaria de programas de negocios internacionales Politécnico Grancolombiano. Administrador de empresas con Magister en comercio internacional.

10. Director de la Escuela de Negocios y desarrollo internacional en el Politécnico Grancolombiano. Economista y Magister en comercio internacional.
} 
que promueven la participación, la comunicación y el trabajo en equipo de los estudiantes. El objetivo de este trabajo es analizar las ventajas y desventajas del uso de esta plataforma en la educación superior.

La metodología a utilizar será cualitativa y descriptiva, ya que se compone de un análisis del modelo de educación virtual y una descripción de las ventajas y desventajas de este método de aprendizaje. Para esto, se realiza una revisión bibliográfica de autores que hayan identificado ventajas y desventajas de este modelo educativo. Así mismo, por medio de una encuesta se identificará las percepciones y nivel de satisfacción de estudiantes del programa de Negocios internacionales de la modalidad virtual. Finalmente, se concluirá con base en los instrumentos usados para la comparación propuesta.

El tema de la educación virtual es muy relevante hoy en día, ya que el avance en la tecnología y la globalización permite ahora que muchas más personas en el mundo puedan acceder a la educación sin trasladarse, manejando de manera flexible su tiempo de estudio; lo único que se necesita es una conexión a Internet, un dispositivo electrónico (tableta, computador, celular inteligente, entre otros), y ante todo la disciplina que debe ser característica de esta modalidad de aprendizaje.

\section{Campus virtual en la educación superior}

Se hace necesario tener claro qué es un campus virtual. Adicional a la definición planteada por Mansilla y Ugliarolo (2013), presentada anteriormente, EuroPACE, una asociación sin ánimo de lucro que agrupa a universidades e instituciones educativas europeas, define un campus virtual como una iniciativa a gran escala, entendiendo la escala como el conjunto de condiciones que incluyen disponer de un mínimo de un 1\% del presupuesto de la institución, plantilla propia, estructura independiente, etc. (Trujillo, 2016).

De igual manera, Trujillo (2016), clasifica los campus virtuales de la siguiente manera: Universidades:

instituciones públicas o privadas que ofrecen titulaciones con registro calificado.

Universidades abiertas:

instituciones con formación a distancia, que están abiertas a todos los habitantes de una zona determinada.

\section{Colegios universitarios:}

instituciones oficiales que no disponen de capacidad para ofrecer títulos de posgrado (instituciones técnicas y tecnológicas).

\section{Escuelas o centros de enseñanza:}

dependiendo del país, instituciones que ofrecen educación terciaria (no formal), pero no a nivel universitario. 
Las funciones del campus virtual son variadas, pero una de las más importantes es promover la comunicación entre los estudiantes y el tutor. Esto se logra por medio de foros (herramienta en donde el tutor plantea una pregunta o problemática y los estudiantes deben opinar acerca de este, justificando su opinión); el foro fomenta el intercambio de ideas entre tutor y estudiantes.

El chat (herramienta que permite al tutor comunicarse de manera simultánea con los estudiantes, bajo unos parámetros de horario y tema determinado), también se ha convertido en una herramienta importante dentro del campus virtual, así como las teleconferencias (herramienta que permite al tutor grabar un video con la explicación de un tema, para que los estudiantes luego puedan visualizarlo), y el correo electrónico ,que es la herramienta más común y utilizada para enviar mensajes y comunicarse entre usuarios del campus.

Es importante resaltar que el buen uso de las plataformas virtuales depende, en gran medida, de la correcta capacitación que tengan los docentes y los estudiantes, lo que requiere tiempo, dedicación y paciencia. El correcto manejo de esta herramienta implica su uso frecuente para estimular el ensayo y error, y su constante actualización para garantizar a los estudiantes la veracidad de la información que contiene la plataforma. Sin embargo, este tipo de herramientas, según Litwin (2005), no considera intereses personales, desafíos cognitivos y alcances o disposición de las tecnologías.

Otro aspecto a tener en cuenta con respecto al uso de las herramientas virtuales de educación es que, para algunos, su uso mejora la condiciones para que se produzca el aprendizaje, mientras que, para otros, las empobrece, en gran medida porque el costo de mantener actualizadas las plataformas virtuales puede llegar a ser alto, o peor todavía cuando no todos tienen acceso debido a falta de infraestructura (Litwin, 2005).

\section{Ventajas y desventajas de las plataformas virtuales en la educación}

La Unesco, en su informe mundial sobre la educación, señala que los entornos de aprendizaje virtuales constituyen una forma totalmente nueva de tecnología educativa, y ofrecen una compleja serie de oportunidades y tareas a las instituciones de enseñanza de todo el mundo (Delgado y Riveros, 2009). La educación virtual, según Durán, Niculcar y Alvarez (2015), presenta como ventaja mayor autonomía e independencia que disfruta el alumno al marcar su propio ritmo de trabajo. De igual manera, mediante el uso de la tecnología en la educación se puede aportar más flexibilidad al diseño del material de estudio, así como propiciar la interacción entre diferentes personas. 
Otras de las ventajas mencionadas por Delgado y Riveros (2009), son:

- $\quad$ El uso de las TIC en educación permite la creación de ambientes educativos motivantes y retadores para la adquisición de conocimientos. Pueden ser utilizadas como una herramienta valiosa para todos los agentes del sistema educativo.

- Las TIC tienen una acción protagónica y significativa en el fenómeno educativo y actúan al unísono sobre el estudiante y sobre el docente, de ahí la necesidad que todo educador tiene de conocer su influencia, consecuencias e implicaciones ante determinada situación educativa, para así utilizarlos de forma óptima y eficiente.

- Las TIC, concebidas como medios informáticos, no son la panacea a los problemas de la educación, ya que deben aplicarse dentro de un contexto didáctico complejo, el cual tome en consideración diversos factores intervinientes en el proceso educativo.

- El uso de recursos informáticos puede ayudar al docente a mejorar la calidad de la enseñanza; además, puede brindar la oportunidad de obtener un mejor aprovechamiento del tiempo libre de sus estudiantes y su aprendizaje de manera activa y consciente. Ofrecen la oportunidad de aprender fuera del aula, en cualquier lugar, en cualquier momento y al propio ritmo.

Dentro de las desventajas que tiene la educación virtual podemos encontrar algunas como:

- $\quad$ El factor cultural, ya que no todos los países tienen la tendencia o la cultura del uso de plataformas virtuales como medio de aprendizaje. Hay personas que aún prefieren asistir a las aulas de clases de manera presencial.

- $\quad$ Otra desventaja es la infraestructura tecnológica con la que cuenta cada país; una infraestructura deficiente puede ir en detrimento del proceso de enseñanza-aprendizaje.

- La capacitación de los docentes en el buen manejo de estas plataformas. Si el docente no está constantemente haciendo un seguimiento a las inquietudes de los estudiantes y respondiendo sus correos y mensajes, la interacción requerida para garantizar la apropiación de conocimientos es apenas parcial.

- $\quad$ Pérdida del control de la clase por parte del docente, especialmente en el tema de la suplantación de estudiantes, plagio y/o copia.

- $\quad$ Escepticismo por parte de los docentes acerca de la manera en que las TIC mejoran los currículos (Sandra Cristina Riascos- Erazo, 2009). 


\section{Implementación de experiencia retadora de aprendizaje}

En el programa de Negocios internacionales del Politécnico Grancolombiano, en los módulos virtuales de logística, globalización y competitividad y negociación internacional, se ha tenido la experiencia de guiar a los estudiantes en su proceso de enseñanza- aprendizaje virtual. En este ámbito es necesario motivar a los estudiantes para que ingresen a la plataforma y para mantener una interacción constante; el docente debe garantizar una comunicación permanente por medio de los canales existentes en la educación virtual. En esta medida, la educación virtual se entiende como el proceso de aprendizaje, no solo para los estudiantes sino para los tutores, ya que es necesario conocer y entender la plataforma virtual para poder garantizar el buen desempeño en el ejercicio de enseñanza.

Igualmente, la creación de los foros se convierte en un reto, ya que debe ser un tema que fomente la participación de los estudiantes y que, al mismo tiempo, sirva para que estos investiguen más acerca del tema planteado. También es necesario que los temas sugeridos lleven a una indagación que vaya más allá de la consulta de contenidos en la red y que evite prácticas como el plagio.

Como apoyo adicional, desde el programa de Negocios internacionales se ha tenido la oportunidad de realizar grabaciones de teleconferencias en inglés y español para incorporarlas a los módulos; esto ha permitido evaluar la manera en que se explican los contenidos, pues se hace constantes auditorías a los contenidos de la plataforma virtual. Las teleconferencias deben ser cortas, pero claras, y deben incluir ayudas didácticas como diapositivas que sirvan de apoyo a la explicación del tutor y que sean una herramienta de consulta permanente para el estudiante.

Por último, para garantizar la interacción permanente se establecen horarios de chat en el transcurso de la semana, con el ánimo de resolver las dudas de los estudiantes frente a algún módulo y guiarlos en los trabajos finales de investigación que deben entregar. El campus virtual del Politécnico Grancolombiano es reconocido en Colombia como uno de los mejores para estudios de pregrado en modalidad virtual. Al final de cada módulo se realiza una evaluación al tutor para medir su compromiso con los contenidos y las herramientas virtuales. El reto de los tutores es mantener la excelente calidad del campus virtual. A continuación se presenta un esquema que representa la forma como se concibe la educación virtual en el Politécnico Grancolombiano: 
Figura 1. Descripción del modelo de interacción en la Educación virtual
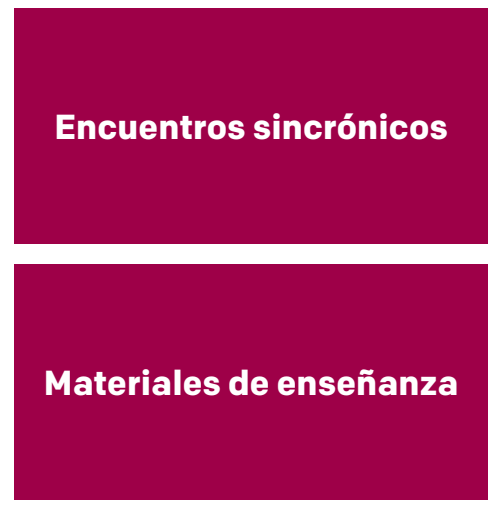

Fuente: elaboración propia (2018).
- Conferencias en las que se profundiza sobre los temas contenidos en los materiales.

- Chat: se usa para garantizar la comunicación permanente entre el tutor y el estudiante.

- Aprendizaje por escenario: lectura fundamental, lectura complementaria, actividades de refuerzo y actividades evaluativas.

- Teleconferencias.

\section{Percepción y nivel de satisfacción de los estudiantes y/o egresados del programa de Negocios internacionales del Politécnico Grancolombiano bajo la modalidad virtual}

Para el presente estudio se indagó sobre la percepción de los estudiantes, con el objeto de identificar el nivel de satisfacción con la educación virtual recibida, al igual que su desempeño y la relación con el desarrollo de competencias para el mundo laboral.

De acuerdo con las preguntas formuladas que provienen del diagnóstico previo que se ha realizado en la sección anterior, y de la revisión de literatura, se usó un método mixto para la recolección de información cualitativa y cuantitativa.

Para medir el nivel de satisfacción y percepción se estableció una escala de uno a cinco:

\section{Muydeficiente 2. Deficiente 3. Regular 4. Bueno $5 x$ 5. Excelente}

En relación con la calidad académica, el 50\% de los estudiantes encuestados consideran que es buena, argumentando que facilita el acceso a las personas que no tienen la disponibilidad de estar presencialmente en una universidad. El acceso se puede hacer desde cualquier lugar y la forma de estudiar los contenidos se puede hacer off-line, es decir, el tutor no necesariamente debe estar conectado en línea para indicar al estudiante qué hacer en su día a día académico.

El 50\% restante considera que es regular o deficiente; esta percepción se atribuye a que la educación presencial recibida en otros niveles, como la secundaria, crea elementos de contraste y expectativas en los estudiantes que sienten que no cuentan con el 
acompañamiento debido. Como se trata de un método de educación tan autónomo, el estudiante suele asumir un rol pasivo en su formación, hecho que afecta su rendimiento y su percepción sobre la calidad.

También argumentan que la comunicación con los tutores en ocasiones se dificulta y no es oportuna; cuestionan, además, la realización de trabajos en grupo, ya que aluden problemas de comunicación que impiden el desarrollo de ejercicios cooperativos de buena calidad.

En contraste con la educación virtual, el 60\% de los estudiantes encuestados percibe como excelente y buena la calidad académica de la educación bajo la modalidad presencial; lo anterior se evidencia en comentarios como: "Aunque la calidad depende mucho del estudiante, el estudiar de forma presencial brinda muchas más posibilidades de profundizar en los temas propuestos para las asignaturas pertinentes y así mismo facilita la solución de dudas, ya que se tiene relación directa con el tutor. Adicionalmente, tienes la oportunidad de interactuar fácilmente con tus compañeros, lo cual facilita el trabajo en equipo y mejora el rendimiento".

El 40\% restante de los estudiantes consideran que la educación de manera presencial es regular, argumentando lo siguiente: “Definitivamente es una experiencia un poco más práctica al estudiar presencial, pues estamos con nuestros compañeros haciendo trabajos presencialmente, sin embargo, las metodologías son un poco aburridas y desgastantes mentalmente (no en todas en clases, pero en la mayoría), debido a que lo que aprendemos, para poder realizar los parciales, siempre hay que memorizar y pienso que así no se logra aprender correctamente; para que podamos recordar las cosas necesitamos ponerlas en práctica, en la vida real y así poder ser competitivos en el mercado laboral'. Esta afirmación da cuenta de la insatisfacción que sienten los estudiantes con los métodos de enseñanza tradicionales.

Gráfico 6. ¿De acuerdo con su experiencia, el tiempo dedicado al trabajo autónomo en la modalidad virtual le ha permitido desarrollar de mejor manera las competencias relacionadas con el módulo o asignatura?

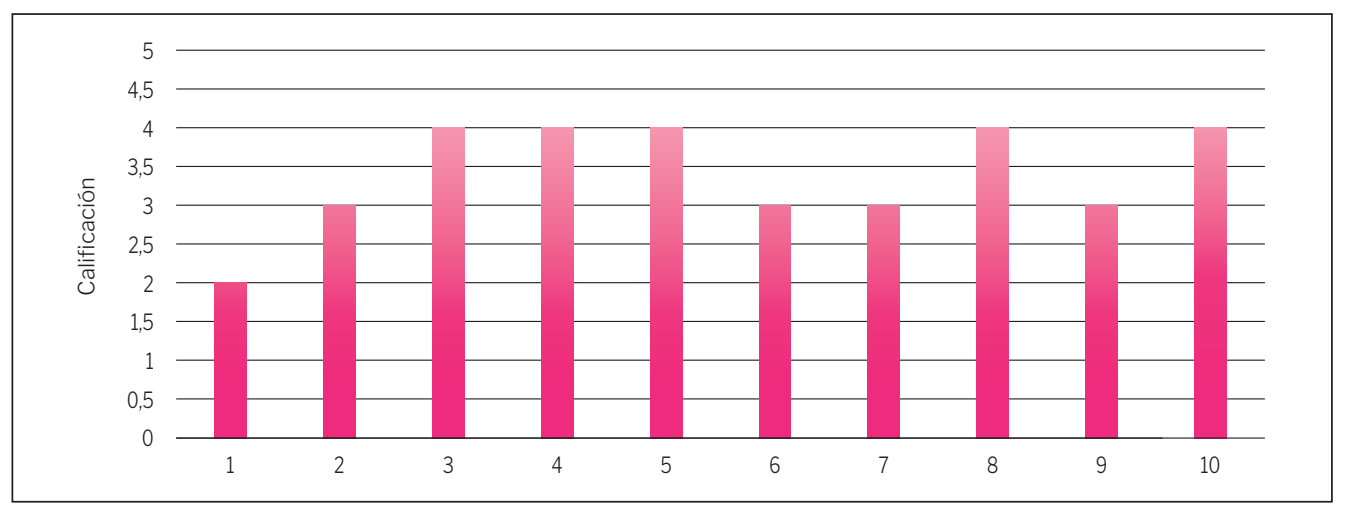

Fuente: elaboración propia (2018). 
Dado que el trabajo autónomo hace parte de los cimientos de la educación virtual, se preguntó a los estudiantes si han podido desarrollar las competencias dispuestas en cada uno de los módulos o asignaturas mediante el trabajo por cuenta propia. Frente a esta pregunta, el 50\% manifestó que ha sido bueno su desarrollo por medio del trabajo autónomo, mientras que el otro $50 \%$ manifiesta que ha sido regular y deficiente. Los comentarios de los estudiantes sobre este particular indican que la falta de tiempo para cumplir de mejor manera las actividades evaluativas, como la lectura del material, dificulta su proceso de aprendizaje autónomo. Esto indica que los estudiantes son conscientes del tiempo que deben destinar para el buen desarrollo de las competencias establecidas por el programa, pero cuentan con medios limitados para hacerlo.

Gráfico 7. ¿Considera que la modalidad virtual le ha permitido desarrollar las competencias necesarias para su ejercicio laboral?

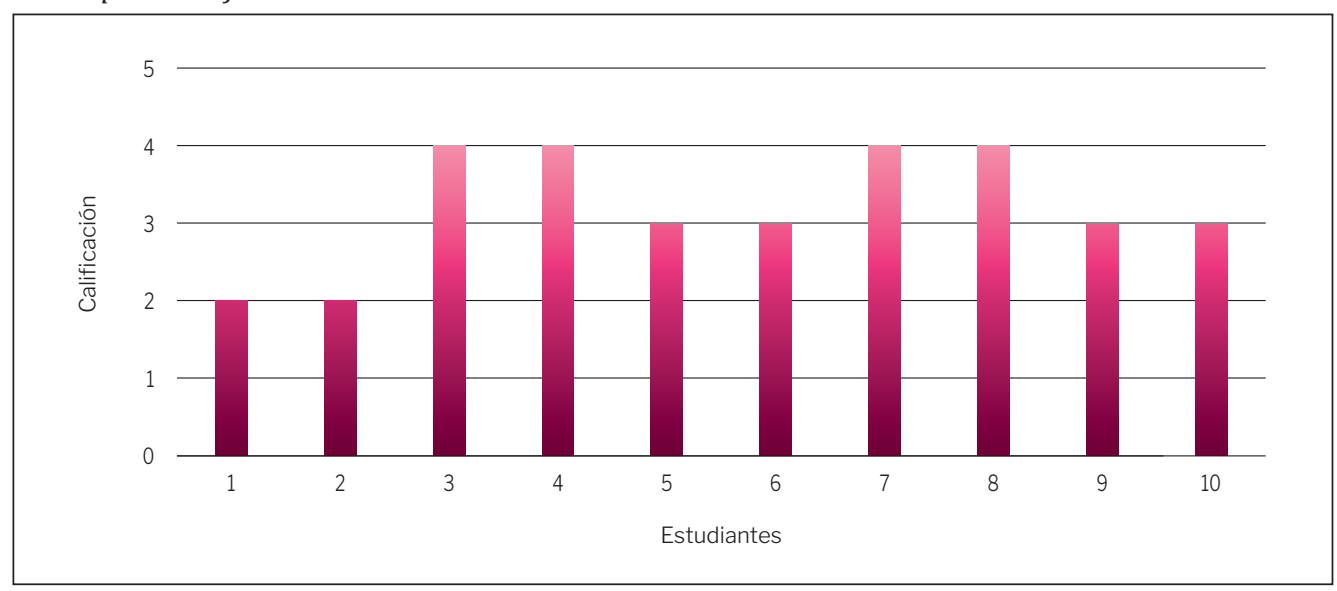

Fuente: elaboración propia (2018).

El 40\% de los estudiantes encuestados consideran que ha sido buena la modalidad virtual para el desempeño de sus competencias, aplicadas en el ejercicio laboral. Algunos de los comentarios fueron:

"Para el caso de negocios, las habilidades financieras, el trabajo de equipoycomercio internacional con un enfoque global han sido fundamentales para mi experiencia profesional".

“Sí, ya que los módulos muestran de manera oportuna y actualizada casos de la vida real".

El 60\% restante considera que es regular, con argumentos tales como:

"Aunque sí se llegan a utilizar algunos de los conceptos aprendidos, es muy poco lo que se recuerda, ya que no se profundizó lo suficiente o no se hizo un estudio a conciencia". 
En la encuesta también se preguntó de manera abierta a los estudiantes sobre los comentarios positivos y negativos que tienen de la modalidad de educación virtual, para lo cual se midieron las frecuencias mediante nubes de palabras:

Ilustración 8. Opinión de los estudiantes en cuanto a los aspectos positivos de la educación virtual

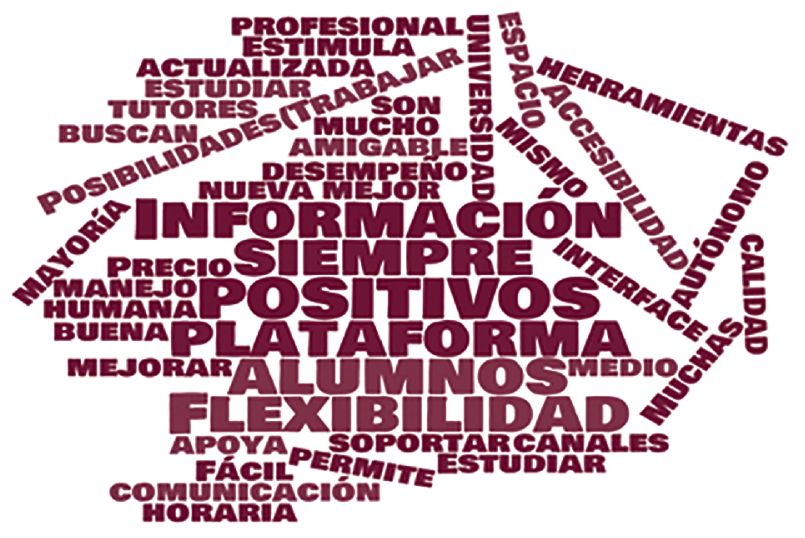

Fuente: elaboración propia (2018).

Dentro de los comentarios positivos, se destaca la flexibilidad horaria de acceso, el precio y las bondades de la plataforma, que en este caso es CANVAS, reconociendo lo amigable de su uso y el material académico.

Ilustración 9. Aspectos negativos señalados por los estudiantes en relación con la educación virtual

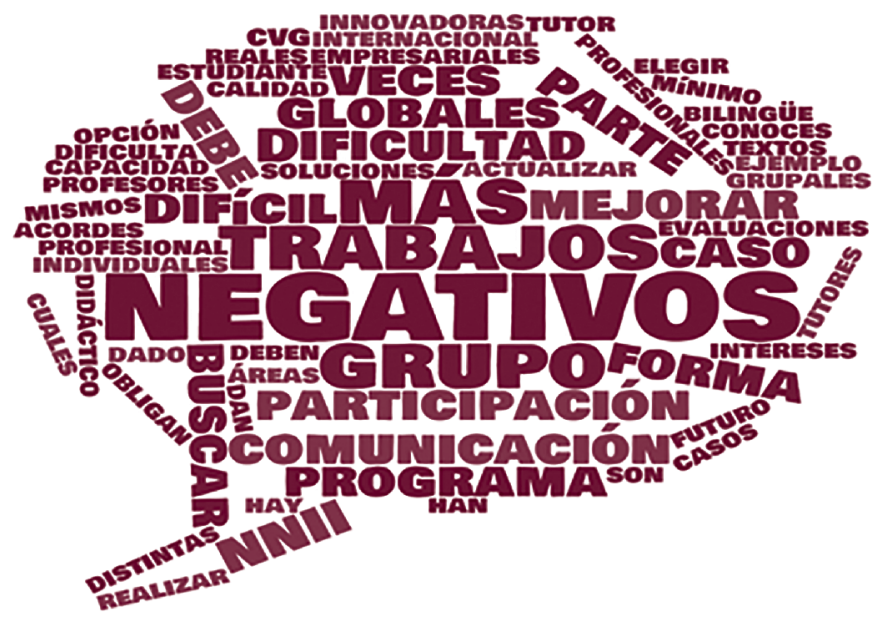

Fuente: elaboración propia (2018). 
Entre los comentarios negativos se destaca los inconvenientes o inconformismo con los trabajos en grupo, argumentando que no es fácil la comunicación, ni la capacidad de trabajo de los compañeros. También argumentan que el acompañamiento por parte del tutor debe ser más expedito y frecuente.

\section{Conclusiones}

Luego de hacer un análisis de este trabajo, se puede concluir que el uso de las tecnologías de la información en la educación puede llegar a ser muy útil, ya que crea nuevas experiencias de comunicación e integración entre estudiantes y profesores. El mundo globalizado brinda la posibilidad de conocer e implementar las nuevas tecnologías como chats, foros, videoconferencias y correos electrónicos para fomentar el aprendizaje y la búsqueda de conocimiento.

Existen ventajas de utilizar las plataformas de educación virtual, tales como evitar el desplazamiento, ya que se puede ingresar desde cualquier lugar del mundo; solo se necesita acceso a Internet y un computador, celular o tableta. De igual manera, el estudiante tiene la posibilidad de aprender a su propio ritmo y manejar su tiempo de la mejor manera.

Sin embargo, es responsabilidad del docente estar capacitado en el uso de las diferentes plataformas virtuales (chats, foros, correo electrónico), y actualizar los contenidos, así como garantizar que los mismos sean didácticos, que faciliten el proceso de aprendizaje de los estudiantes, que promuevan el desarrollo de competencias empresariales y que puedan ser reutilizados.

De igual manera, los estudiantes deben tomar conciencia de la importancia de la disciplina en este tipo de educación, ya que, al ser virtual, requerirá de una mayor autonomía, manejo del tiempo, planeación, disciplina y automotivación.

De acuerdo con las encuestas realizadas, los estudiantes sí perciben la educación virtual como una herramienta que facilita el acceso a la educación, ya que no es obligación estar de manera presencial en un salón de clases, y el material se puede consultar en cualquier momento; pero también son conscientes que, para lograr las competencias educativas, deben dedicarle tiempo y ser organizados.

Como aclaran Mansilla y Ugliarolo (2013), la tecnología no implica una buena enseñanza, sino que debe considerarse como una herramienta adicional para mejorar, estimular y potenciar la misma. El éxito de un proceso de aprendizaje virtual no va a depender solo del uso que se haga de las tecnologías de la información, sino de los contenidos pedagógicos y motivadores que se elaboren, que promuevan el trabajo colaborativo y la reflexión y, sobre todo, en los que se tenga la misma exigencia que con la educación presencial. 


\section{Referencias}

Barba, C. F. (2015). Internet en educación superior. Revista de la educación superior, 177-182.

Barrios, A. H., y Uribe, A. C. (2017). Autorregulación del aprendizaje en la educación superior en Iberoamérica: una revisión sistemática. Revista latinoamericana de Psicología, 146-160.

Campillo-Serrano, C. D., Morales-Gordillo, N., Trejo-Marquez, H. D., Martínez, J. L., Medina, I. K., Gallegos-Cázares, R., ... Magallanes, C. R. (2012). La educación en línea: una metodología flexible para formación de residentes de Psiquiatría. Elsevier, 87-93.

Cerezo, R., Bernardo, A., Esteban, M., Sánchez, M., y Tuero, E. (2015). Programas para la promoción de la autorregulación en educación superior: un estudio de la satisfacción diferencial entre metodología presencial y virtual. European Journal of education and phsycology, 30-36.

Delgado, M., y Riveros, X. A. (2009). Uso de las TIC en educación, una propuesta. OMNIA, 58-77.

Dios, J. G., y Bandera, F. H. (2017). Continuum: el poder del aprendizaje virtual y la Web 2.0 en la formación médica en Pediatría. Tres años de experiencia. Educación Médica, 1-9.

Durall, E., Gros, B., Maina, M., Johnson, L. y Adams, S. (2012). Perspectivas tecnológicas: educación superior en Iberoamérica 2012-2017. Austin, Texas: The New Media Consortium.

Durán, R., Estay-Niculcar, C., y Álvarez, H. (2015). Adopción de buenas prácticas en la educación virtual en la educación superior. Aula Abierta, 77-86.

Litwin, E. (2005). Tecnologías educativas en tiempos de Internet. Buenos Aires: Amorrortu Editores.

Mansilla, D. S., Muscia, G. C., y Ugliarolo, E. A. (2013). Campus Virtual y Facebook en el ámbito universitario. ¿Enemigos o aliados en los procesos de enseñanza y aprendizaje? Educación química, 255-259.

Nacional, M. d. (09 de julio de 2009). Ministerio de Educación Nacional. Obtenido de https://www.mineducacion.gov.co/1759/w3-article-196492.html

Nahón, A. E. (2017). Aportaciones al proceso horizontal de transversalización de la Educación a Distancia en las instituciones de educación superior. Revista de la educación superior, 57-69.

Pinto, L. (2006). Tecnología educativa para innovar en el trabajo pedagógico. Didáctica, Innovación y multimedia, 1-7.

Rodríguez, J. S. (2009). Plataformas de enseñanzas virtuales para entornos educativos. 
Pixel. Bit. Revista de Medios y comunicación, 217-233.

Saavedra, S. M. (2014). Perfeccionamiento docente virtual: Una experiencia con tutores/as. Perfiles Educativos, 180-194.

Sampieri, R. H. (2003). Metodología de la Investigación. Mexico: McGraw Hill.

Silvio, J. (2006). Educación a distancia y presencia: diferencias en los componentes cognitivo y motivacional de estudiantes universitarios. RIED. Revista Iberoamericana de educación a distancia, 65-75. 